\title{
Psychopharmacological options for adult patients with anorexia nervosa: the patients' and carers' perspectives integrated by the spectrum model
}

\author{
Mario Miniati, * and Donatella Marazziti
}

Dipartimento di Medicina Clinica e Sperimentale, Section of Psychiatry, University of Pisa, Pisa, Italy

Received 6 June 2017; Accepted 24 July 2017; First published online 9 October 2017

Key words: Anorexia nervosa, randomized controlled trial, psychopharmacological treatment, spectrum model, body dysmorphic disorder.

The present letter is in response to a comment on the authors' paper entitled "Psychopharmacological Options for Adult Patients with Anorexia Nervosa," published in CNS Spectrums.

\section{Dear Editor,}

We read with great interest the letter by Himmerich et al., "Psychopharmacological Options for Adult Patients with Anorexia Nervosa: The Patients' And Carers' Perspective,"1 commenting on our paper recently published in CNS Spectrums. ${ }^{2}$ We found noteworthy their proposal of taking a deeper look at the limitations that anorexia nervosa (AN) patients might have regarding drug treatments, especially those that might increase body weight.

The authors focused on two main problems: (1) the poor compliance of $\mathrm{AN}$ patients might derive from scarce educational support and from clinicians' underestimation of patients' subjective experience of the illness; and (2) patients' perceptions of drugs as "unspecific" for AN signs and symptoms and their concerns about weight gain. We agree with Himmerich et al. ${ }^{1}$ that drugs are often described by patients as reducing anxiety and helpful with sleep problems and depressive symptoms but are ineffective in terms of the psychopathological core of $\mathrm{AN}$-namely, their self-perception of body dysmorphia.

Himmerich et al. ${ }^{1}$ summarized the results from a quality improvement (QI) project on a specialist unit for eating disorders (EDs) at the South London and Maudsley National Health Service Foundation Trust.

* Address for correspondence: Mario Miniati, Dipartimento di Medicina Clinica e Sperimentale, Section of Psychiatry, University of Pisa, 57 Via Roma, Pisa, 56100, Pisa, Italy.

(Email: mario.miniati@psico.med.unipi.it)
They highlighted the distance between patient's subjective perception of the illness and that of caregivers regarding both pharmacological options and therapeutic goals. We strongly agree with the authors that systematic adoption of outcome measures focused on effectiveness, safety, and subjective experience of care from a patient's perspective should be considered for future randomized controlled trials (RCTs). Along these lines, more than 10 years ago $(2002)^{3}$ we suggested the use of a "spectrum assessment" as a phenotypic dimensional description of the most important psychiatric disorders, including EDs, directed toward the full extent of the signs, symptoms, and traits experienced by the patient over a lifetime. The rationale for this model was actually twofold: (1) to assess and better understand the course of AN symptomatology, and (2) to highlight the signs and symptoms that patients consider relevant to their "subjective experience of illness." The phenotypic description of AN within the "spectrum assessment method" can provide a tailored picture of the single patient, and when patients complete the spectrum assessment, they are likely to report feeling that their symptoms are better understood by their clinicians.

We proposed this approach in several studies, mainly with respect to mood and anxiety disorders. ${ }^{4}$ In the psychopathological area of EDs, we constructed and validated an interview and a self-rating instrument, the Anorexic-Bulimic Spectrum Questionnaire (ABS-SR), within the framework of an Italian multicenter study. ${ }^{5}$ The ABS-SR encompassed nine psychopathological domains: (1) attitudes and beliefs; (2) weight history; (3) self-esteem and satisfaction; (4) phobias; (5) avoidant and compulsive behaviors; (6) weight maintenance; (7) eating dyscontrol; (8) associated features and consequences; and (9) interference and level of insight.

The second point of discussion raised by Himmerich et $a .^{1}$ is related to the difficulties experienced by 
patients in taking drugs that are mainly targeted at anxiety and mood symptoms, such as selective serotonin reuptake inhibitors (SSRIs), or drugs that are perceived as "dangerous" because of feared side effects or weight gain, common with both antipsychotics and SSRIs. Again, we agree with the authors that studies focused on increasing weight had little success, because weight gain is the clinician's goal, not the patient's. We also agree on the proposal for a scientific exploration of patients' goals as part of future RCTs. In line with this idea, we believe that the role of dopamine 1 and 2 receptor antagonists should be reconsidered in a new light, and they should be utilized for the more severe forms of $\mathrm{AN}$, when body image distortion reaches delusional intensity and compliance is limited by poor insight. However, the evidence in this field is still limited. The impact on delusional fear of weight gain or on body image distortion has usually been disregarded. In view of this, the rationale for administration of antipsychotics has been more closely associated with psychomotor hyperactivity control and with induction of weight gain, thus reinforcing the distance between the goals of patients and clinicians. Conversely, in line with Himmerich et al., ${ }^{1}$ we propose an integrated approach that considers the systematic exploration of a patient's goals as clinically relevant. We also propose a more refined phenotypic description of the different (and subjectively perceived) forms of AN (with the "spectrum model assessment") and the adoption of a new rationale for the use of "old drugs" (such as the antipsychotics) that focus on body image distortion rather than on sedation or weight gain.

\section{Disclosures}

Dr. Miniati and Dr. Marazziti have nothing to disclose.

\section{REFERENCES:}

1. Himmerich H, Joaquim M, Bentley J, et al. Psychopharmacological options for adult patients with anorexia nervosa: the patients' and carers' perspective. CNS Spectr. 2017. doi: 10.1017/ S1092852917000529. In press.

2. Miniati M, Mauri M, Ciberti A, Mariani MG, Marazziti D, Dell'Osso L. Psychopharmacological options for adult patients with anorexia nervosa. CNS Spectr. 2016; 21(2): 134-142.

3. Cassano GB, Frank E, Miniati M, et al. Conceptual underpinnings and empirical support for the mood spectrum. Psychiatr Clin North Am. 2002; 25(4): 699-712.

4. Miniati M, Rucci P, Frank E, et al. Sensitivity to change and predictive validity of the MOODS-SR questionnaire, last-month version. Psychother Psychosom. 2009; 78(2): 116-124.

5. Mauri M, Borri C, Baldassari S, et al. Acceptability and psychometric properties of the Structured Clinical interview for Anorexic-Bulimic Spectrum (SCI-ABS). Int J Methods Psychiatr Res. 2000; 9(2): 68-78. 